\title{
Effect of conditioned medium of mesenchymal stem cells on the in vitro maturation and subsequent development of mouse oocyte
}

\author{
B. Ling ${ }^{1,2}$, D.Q. Feng' 2 , Y. Zhou' ${ }^{2}$, T. Gao ${ }^{1}$, H.M. Wei ${ }^{3}$ and Z.G. Tian ${ }^{3}$ \\ ${ }^{1}$ Department of Obstetrics and Gynecology, ${ }^{2}$ Anhui Province Key Laboratory of Molecular Medicine, \\ Anhui Provincial Hospital Affiliated to Anhui Medical University, Hefei, China \\ ${ }^{3}$ Institute of Immunology, Hefei National Laboratory for Physical Science at Microscale and School of Life \\ Science, University of Science and Technology of China, Hefei, China
}

Correspondence to: B. Ling, Department of Obstetrics and Gynecology, Anhui Provincial Hospital, Anhui Medical University, 17 Lu-Jiang Road, Hefei 230001, China

Fax: +86-551-228-2121. E-mail: lingbin.ling@gmail.com

Mesenchymal stem cells (MSCs) secrete a variety of cytokines and growth factors in addition to self-renewal and multiple forms of differentiation. Some of these secreted bioactive factors could improve meiotic maturation in vitro and subsequent embryo developmental potential. The aim of the present study was to determine whether in vitro maturation (IVM) of mouse oocyte with or without cumulus cells could be improved by contact with conditioned medium (CM) of MSCs as well as the efficiency of CM to support follicular growth and oocyte maturation in the ovarian organ of mice cultured on soft agar. The developmental potential of matured oocyte was assessed by blastocyst formation after in vitro fertilization (IVF). Germinal vesicle stage oocytes with or without cumulus cells were subjected to IVM in either CM, Dulbecco's modified Eagle's medium (DMEM), $\alpha$-minimum essential medium ( $\alpha-M E M)$ or human tubal fluid (HTF). Approximately 120 oocytes were studied for each medium. CM produced a higher maturation rate $(91.2 \%)$ than DMEM (54.7\%), $\alpha$-MEM (63.5\%) and HTF (27.1\%). Moreover, CM improved embryo development to blastocyst stage significantly more than DMEM and HTF (85 vs $7 \%$ and $41.7 \%$, respectively) but there was no significant difference compared with $\alpha$-MEM (85 vs $80.3 \%$ ). The behavior of cortical granules of IVM oocytes cultured in CM revealed cytoplasmic maturation. Moreover, $\mathrm{CM}$ also supported preantral follicles growth well in organotypic culture on soft agar resulting in the maturation of $60 \%$ of them to developmentally competent oocytes. The production of estrogen progressively increased approximately 1-fold every other day during organ culture, while a dramatic 10-fold increase in progesterone was observed 17 $\mathrm{h}$ after human chorionic gonadotropin stimulus at the end of culture. Thus, CM is an effective medium for preantral follicle growth, oocyte maturation, and sequential embryo development.

Key words: Mesenchymal stem cells; In vitro maturation; Follicular development; Mouse oocyte development

Research supported by the China National Natural Science Foundation (\#34071805), and the Science Research Foundation from Anhui Provincial Hospital.

Received December 13, 2007. Accepted October 17, 2008

\section{Introduction}

Technologies to stimulate oocyte growth and development in culture can provide valuable experimental tools for studies of the mechanisms governing oocyte development as well as support practical clinical, agricultural, and zoo- logical applications. During recent years, many attempts have been made to grow immature oocytes in vitro (1-3). It has been established that the culture conditions used for in vitro maturation (IVM), which include different media, type and hormone content, as well as the presence of cumulus cells, can significantly influence maturation rates and sub- 
sequent embryo development (1,4-6). Furthermore, during ovarian follicular development, several growth factors and cytokines act as intra-ovarian regulators in vivo, and in the follicle the action of gonadotrophins is modulated by locally produced paracrine and autocrine growth factors $(7,8)$.

It is known that oocytes need to undergo cytoplasmic maturation as well as nuclear maturation to become able to support successful fertilization and embryo development $(9,10)$. However, a premature resumption of meiosis without adequate cytoplasmic maturation frequently occurs in IVM. Nuclear maturation can be easily identified with germinal vesicle breakdown (GVBD) and polar body extrusion, while cytoplasmic maturation is hard to identify due to the absence of a direct marker.

Mesenchymal stem cells (MSCs) are adult stem cells usually obtained from bone marrow stroma, which are attractive candidates for cell-based therapeutic strategies, primarily because of their intrinsic ability to self-renew and undergo multipotential differentiation as well as being amenable to genetic manipulation (11-13). Given the correct stimuli and local environment, they develop into various cell types in vitro and regenerate tissues in vivo. It is also known that MSCs secrete a variety of cytokines and growth factors, such as MCP-1, VEGF-A, EGF, FGF-2, IL-6, LIF, TGF-B $(12,14)$.

Since it is known that cytokines and growth factors stimulate meiotic progress and the processes associated with IVM, the aim of the present study was to determine whether IVM with cumulus cells in mice could be improved by conditioned medium (CM), and the efficiency of $\mathrm{CM}$ for in vitro follicular growth of organotypic culture on soft agar. The parameters analyzed were follicular growth, meiotic maturation of oocytes, hormone production, cortical granules (CG) distribution, and development of embryos constructed by in vitro fertilization (IVF), to assess the rate of meiotic maturation and quality of oocytes.

\section{Material and Methods}

Animals
Kunming mice, originally derived from ICR/CD1 (Insti-
tute for Cancer Research/Caesarean Derived-1, closed
colony of Swiss mice), were housed in a controlled envi-
ronment $\left(20-25^{\circ} \mathrm{C}\right)$, humidity $(40-60 \%)$ and a $12-\mathrm{h}$ light
cycle (lights on from $8: 00$ to $20: 00)$. Ovaries and oocytes
were obtained from F1 (4 weeks old) females. Sperm for
IVF in studies of preimplantation development were from
F1 (6-8 weeks old) males. Animal care and handling were
conducted in accordance with policies on the care and use
of laboratory animals of the Ethics Committee of Anhui
Provincial Hospital Affiliated to Anhui Medical University.
Mesenchymal stem cell isolation and harvesting conditioned medium

The mice were deeply anesthetized with chloral hydrate and decapitated. Bone marrow was collected by flushing femurs and tibias with Dulbecco's modified Eagle's medium (DMEM; Gibco, USA). Mononuclear cells were harvested by $60 \%$ Percoll (Amersham Bioscience, Sweden) separation (density $1.077 \mathrm{~g} / \mathrm{mL}, 800 \mathrm{~g}$ for $20 \mathrm{~min}$ ) and washed twice (270 $\mathrm{g}$ for $10 \mathrm{~min}$ ) in DMEM. Cells were then incubated in complete medium composed of DMEM, $10 \%$ fetal calf serum (FCS; Gibco), and penicillin/streptomycin (100 U/mL and $100 \mathrm{mg} / \mathrm{mL}$, respectively) at a cell density of $5 \times 10^{6}$ cells $/ \mathrm{mL}$. After 3 days, nonadherent cells were removed by two to three washes with 1 X PBS and adherent cells further cultured in complete medium until $90 \%$ confluent. Surface markers of MSCs were analyzed with fluorescence-activated cell sorter. The following cell surface markers CD11b-, CD34-, CD44+ ${ }^{+}$, CD105 were identified. They are consistent with the literature consensus concerning the markers of MSCs (13). Adherent cells were trypsinized and seeded at a density of $3 \times 10^{5}$ cells $/ \mathrm{mL}$ for further expansion. After the medium was changed once, the supernatant was collected at $48 \mathrm{~h}$, denoted $\mathrm{CM}$ of MSCs and filtered through a $0.2-\mu \mathrm{m}$ membrane for immediate use.

Collection and in vitro maturation of immature germinal vesicle oocytes

Immature germinal vesicle (GV) oocytes were collected from ovaries of 4-week-old female mice that were injected with $5 \mathrm{IU}$ pregnant mare serum gonadotrophin (PMSG) $46 \mathrm{~h}$ previously. Using a stereomicroscope (Olympus, Japan), large and small antral follicles were punctured with a 27-G needle in a Hepes-buffered M2 medium (Sigma, USA). Based on cumulus characteristic, two categories of cumulus-oocyte complexes (COCs) were selected for IVM: type 1, COCs with a complete, compacted, and multilayered cumulus (Figure $1 \mathrm{~A}$ and $\mathrm{B}$ ); type 2, the cumulus was completely or, in great part, absent or expanded (Figure 1C and D). After washing in HEPESbuffered M2 medium, the two types of COCs were subjected to IVM in one of four media: $\alpha$-minimum essential medium ( $\alpha$-MEM) (Gibco), DMEM supplemented with $10 \%$ FCS, human tubal fluid (HTF; LifeGlobal, USA) using $0.4 \%$ BSA as a protein source, and CM. Oocyte maturation was evaluated at $8,16,24,32,40$, and $48 \mathrm{~h}$.

\section{Organotypic culture on soft agar}

Six hours after attaching on 6 -well culture plates with a density of $1 \times 10^{5}$ cells $/ \mathrm{mL}$, MSCs were coated with $4 \mathrm{~mL}$ $0.4 \%$ agar (Sigma, USA) in CM (5\% agar diluted with CM) 
supplemented with $1.5 \mathrm{IU} / \mathrm{mL}$ PMSG. The ovaries of 4week-old mice were freed from connective tissues under a stereomicroscope and cut into $500-\mu \mathrm{m}$ thick slices. Five slices were placed on soft agar in each well. The spare soft agar in the vicinity of the ovary section was removed and the vacancy filled with $2 \mathrm{~mL} \mathrm{CM}$ containing $1.5 \mathrm{IU} / \mathrm{mL}$ PMSG. Half of the medium was renewed every other day.

At day 6 of culture, the medium was replaced by $\mathrm{CM}$ supplemented with $1.5 \mathrm{IU} / \mathrm{mL}$ human chorionic gonadotropin (hCG) to induce final oocyte maturation. After $17 \mathrm{~h}$, COCs were released from antral follicles and incubated in $\mathrm{CM}$ for $3 \mathrm{~h}$, then collected using a Pasteur pipette. The oocytes were scored for maturation and fertilized in vitro after removal of cumulus cells.

\section{In vitro fertilization and embryo development}

For the assessment of the developmental potential of oocytes that had undergone GVBD or reached the MII stage via IVM, the oocytes free of cumulus cells were subjected to IVF experiments. Sperm was collected from cauda epididymides of the males (6-8 weeks old), capacitated for 1 to $1.5 \mathrm{~h}$, and diluted in HTF to a final concentration of 0.7 to $1.3 \times 10^{6} \mathrm{sperm} / \mathrm{mL}$. After insemination, the oocytes were cultured in Chatot-Ziomek-Bavister medium (CZB) supplemented with $0.4 \%$ BSA for $96 \mathrm{~h}$ to examine their subsequent development.

As controls, in vivo matured MII oocytes were retrieved from the oviducts of superovulated females (4 weeks old) following injection with 5 IU PMSG and then 5 IU hCG $48 \mathrm{~h}$ later. Approximately $15 \mathrm{~h}$ after hCG injection, cumulusenclosed oocytes were collected from the oviductal ampullae, and released from the cumulus cells by treatment with $0.1 \%$ hyaluronidase (Sigma) in M2 medium. They were subsequently fertilized in vitro for evaluation of their developmental competence.

\section{Staining of CG and confocal microscopy}

To investigate the quality of IVM conditions, oocytes matured in $\mathrm{CM}$ and sequentially fertilized in vitro were stained for CG. Oocytes in M2 were treated with $0.5 \%$ (w/ v) protease $E$ (BioShare, Germany) for 3-5 min to remove the zona pellucida and fixed in $3.7 \%$ paraformaldehyde in $\mathrm{M} 2, \mathrm{pH} 7.4$, for $30 \mathrm{~min}$. After washing three times in blocking solution (M2 containing $0.3 \%$ BSA and $100 \mathrm{mM}$ glycine), oocytes were permeabilized with $0.1 \%$ Triton $X$ 100 in M2 for 5 min. After washing twice in blocking solution, oocytes were incubated in M2 containing $100 \mu \mathrm{g} /$ $\mathrm{mL}$ fluorescein isothiocyanate-lens culinaris agglutinin (Sig$\mathrm{ma}$ ) for $30 \mathrm{~min}$. Oocytes were then washed three times (5 min each) in M2 containing $0.3 \%$ BSA and $0.01 \%$ Triton X100 and stained with $10 \mu \mathrm{g} / \mathrm{mL}$ propidium iodine (Sigma) for $10 \mathrm{~min}$ to visualize chromosomes. The samples were examined with a laser-scanning confocal microscope (LSM510, Zeiss, Germany).

\section{Assessment of hormone production in in vitro organotypic culture}

From day 1, the culture medium was removed and stored at $-20^{\circ} \mathrm{C}$ for hormone analysis. Estradiol and progesterone were measured using radioimmunoassays from Clinical Assays (Union Medical and Pharmaceutical Technology Tianjin Ltd., China) with analytical sensitivity and precision (CV\%) of $1.4 \mathrm{pg} / \mathrm{mL},<10 \%$ and $0.05 \mathrm{ng} / \mathrm{mL}$, $<5 \%$, respectively. Both assays were validated for the use of culture medium as the sample.

\section{Statistical analysis}

Four treatment groups, DMEM, a-MEM, HTF, and CM, consisted of six replicate experiments. Each experiment started with approximately 120 oocytes. Variations between intra-experimental measurements are reported as \pm
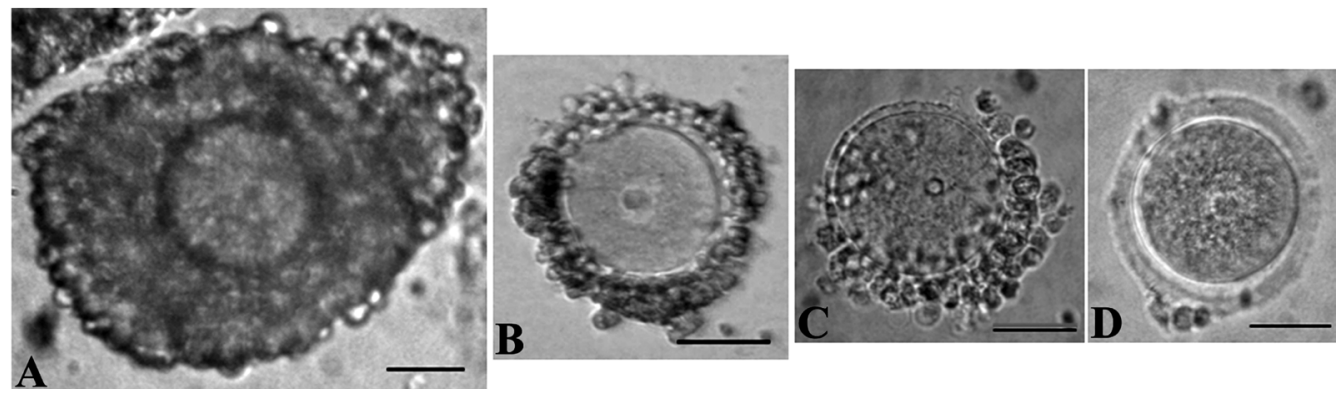

Figure 1. Immature germinal vesicle oocytes, isolated from 4-week-old mice $46 \mathrm{~h}$ after $5 \mathrm{IU}$ pregnant mare serum gonadotrophin injection, were classified by the layers of cumulus cells. Type 1 ( $A$ and $B$ ): enclosed with more than one layer of compact cumulus cells; type 2 (C and D): naked/partially naked. Bars $=50 \mu \mathrm{m}$. 
SEM. For evaluation of the differences between treatment groups, data were subjected to the Fisher protected least significant difference test and ANOVA. A probability of $\mathrm{P}<$ 0.05 was considered to be statistically significant.

\section{Results}

\section{IVM of oocytes with different media}

A high maturation rate of two types of oocytes was obtained when CM was used as the medium. Although IVM in $\mathrm{CM}$ resulted in the excellent meiotic competence of immature oocytes, it led to an apparent time-dependent process of oocyte aging with prominent manifestations of being sensitive to activating stimuli, the onset of anaphase II, and partial exocytosis of CG (data not shown), as described by Miao et al. (15). Hence, the optimal duration of IVM in CM for type 1 was $24 \mathrm{~h}$ while for type 2 oocytes it was $16 \mathrm{~h}$. The maturation rate in $\mathrm{CM}$ was significantly greater than that in DMEM, a-MEM and HTF $(P<0.01)$ compared within types. However, no significant differences were demonstrable between type 1 and type 2 oocytes for each culture medium except HTF. In this case, the maturation rate was significantly greater for oocytes of type 1 (with complete cumulus) than those of type 2 (Figure 2).

Follicular growth in organotypic culture

At day 2, all surviving follicles began to initiate growth. The preantral follicles showed a significant increase in

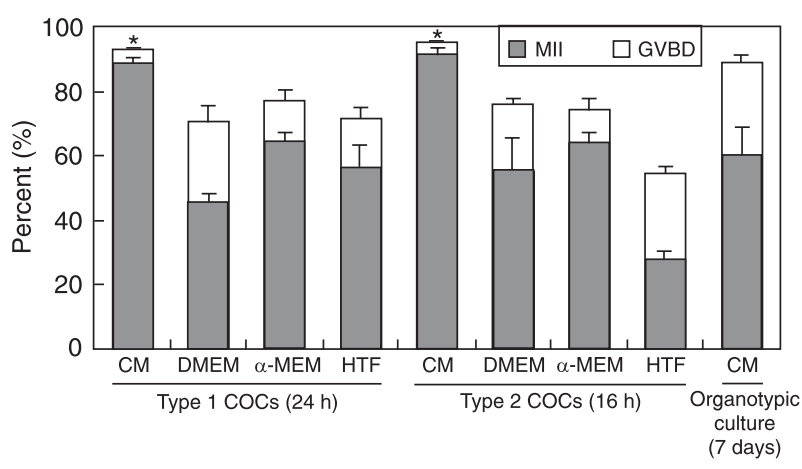

Figure 2. Nuclear maturation of two types of mouse oocytes after in vitro culture in CM, DMEM, a-MEM and HTF, respectively, and the oocytes from follicles after organotypic culture and matured in $\mathrm{CM}$. The bars represent the combined results of six replicate experiments. Each experiment started with approximately 120 oocytes in each group. Asterisks at the top of the columns denote significant differences $(P<0.01)$ among treatment groups within types. MII = metaphase II; GVBD = germinal vesicle breakdown; $\mathrm{CM}=$ mesenchymal stem cell conditioned medium; DMEM = Dulbecco's modified Eagle's medium; a-MEM = a-minimum essential medium; HTF = human tubal fluid; COCs = cumulus-oocyte complexes. diameter, which resulted in some follicles protruding from the surface of the cultured organ. The maximum diameter reached approximately $100 \mu \mathrm{m}$. However, in these follicles, only 1-2 layers of granulosa cells were present. The granulosa cells had rather tight intercellular contact (Figure $3 \mathrm{~A}$ ). With further 24-h culture, the granulosa cells began to grow rapidly and multiple layers surrounded the oocyte. At the end of culture, all surviving follicles showed translucent areas within the granulosa cell mass around the oocyte after $17 \mathrm{~h}$ of hCG stimulus (Figure 3B). To evaluate maturation, $88 \%$ of the oocytes from these antral follicles underwent GVBD, and $60 \%$ of them extruded the first polar body (Figure 2).

Follicle degeneration occurred at any time of culture and was characterized by either failure of the granulosa cells to multiply or oocyte release (Figure 3C). Some of the oocytes, released from follicles without full growth, cultured on soft agar containing CM for a further 16 to $24 \mathrm{~h}$ resulted in the first polar body extrusion (Figure 3D). However, these oocytes were designated as nonsurviving and were eliminated from the experiment.
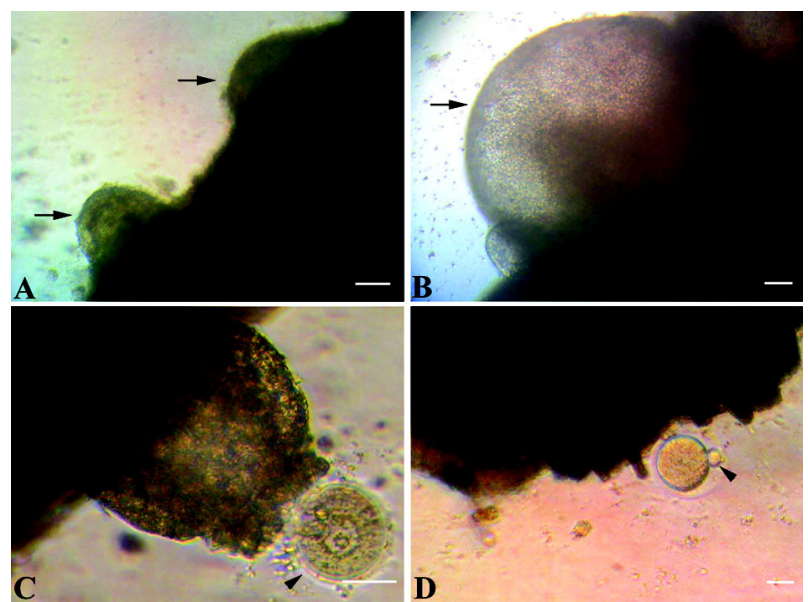

Figure 3. Follicular growth in ovarian organ cultured on soft agar. $A$, At day 2, the preantral follicles began to initiate growth, and protruded from the surface of cultured organ due to increase in follicle diameter (arrows). The follicles with a diameter of 80-100 $\mu \mathrm{m}$ contained one to two layers of compact granulosa cells and an immature oocyte. $B$, At the end of culture, follicles showed pronounced outgrowth of the granulosa cells and translucent areas (antral-like cavities) within the granulosa cell mass around the oocyte, indicating the formation of antral follicles (arrow). $C$, A small fraction of follicles showed no apparent growth, characterized by failure of the granulosa cells to multiply, and oocyte release (arrowhead). $D$, Some of the oocytes, released from follicles without full growth presented a first-polar body extrusion (arrowhead) when cultured on soft agar containing conditioned medium for a further 16 to $24 \mathrm{~h}$. These oocytes were discarded. Bars $=50 \mu \mathrm{m}$. 
Developmental competence of IVM oocytes following IVF

The developmental competence of IVM oocytes was assessed by IVF and subsequent embryo culture to the blastocyst stage. A large fraction of the oocytes (82.9\%) that underwent GVBD cleaved to the two-cell stage after insemination when the oocytes were matured in CM compared with $30 \%$ matured in DMEM and $58.5 \%$ matured in HTF $(P<0.01)$. There was no significant difference of oocytes cleaved to the two-cell stage between CM (82.9\%), a-MEM (84.2\%), and in vivo matured $(89 \%)$, but it was greater than the oocytes isolated from in vitro cultured ovarian organ and matured in $\mathrm{CM}(63 \%$; $\mathrm{P}<0.01$; Figure 4).

The percent of blastocyst derived from CM, a-MEM, maturation in vivo and organotypic culture was not significantly different: $85,80.3,87.5$, and $82.4 \%$, respectively $(P$ $>0.05)$. The percent completing the two-cell stage to blastocyst transition was less in HTF and DMEM than in CM: 41.7 and 7 vs $85 \%(P<0.01)$. There was a 12-fold difference of CM over the DMEM control.

The oocytes described above for IVF were from category 1. The developmental competence of oocytes of type 1 IVM in DMEM, a-MEM, or HTF, was superior to those of type 2 (data not shown) although the maturation rate was not significantly different between the two types. However, when CM was selected as the maturation medium, no significant difference in development was exhibited between the two type cells.

Changes in the localization of CG during oocyte IVM and IVF

The localization of CG was determined by confocal microscopy in IVM oocytes, which were at maturation stages of GV, GVBD, and MII, respectively, during culture in $\mathrm{CM}$, and sequentially in IVF embryos. At stage GV, almost all CG were located in the cortex with few observed in the inner cytoplasm (Figure 5A). At stage GVBD, the number of $C G$ in the cortex decreased in the vicinity of the $\mathrm{MI}$ spindle, and then the first CG-free domain was well formed (Figure 5B). As the oocytes matured into MII at the end of IVM, a large second CG-free domain formed, and partial CG exocytosis occurred in some MII oocytes (Figure $5 \mathrm{C}$ ). Full-CG exocytosis was observed in the in vitro fertilized oocytes (Figure 5D).

Hormone production in in vitro organotypic culture

The production of estradiol progressively increased during culture (Figure 6A). The basal progesterone production (before hCG stimulus) remained low in this organotypic culture. The production of progesterone increased dramatically $17 \mathrm{~h}$ after stimulation with $1.5 \mathrm{IU} / \mathrm{mL}$

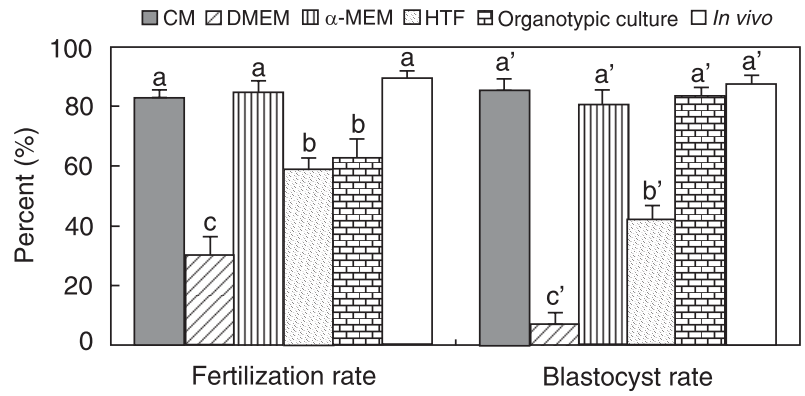

Figure 4. Developmental competence of mouse oocytes matured in vitro and oocytes grown in vitro from preantral follicles after in vitro fertilization. The matured oocytes included those that had progressed to both MI and MII. Each treatment group consisted of six replicates. Groups with different letters (a-c and $\left.a^{\prime}-c^{\prime}\right)$ for each end point were significantly different $(P<0.05)$. See legend to Figure 2, for abbreviations.
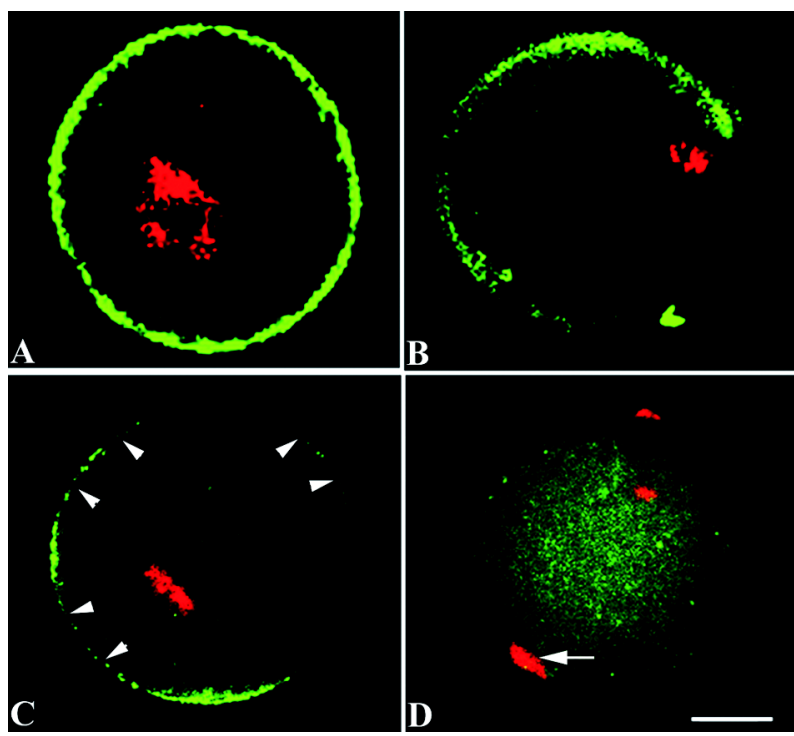

Figure 5. Laser scanning confocal microscopic images of cortical granules (CG; green) and chromatin (red) in mouse oocytes at different stages of maturation or activation. $A$, Oocyte at the germinal vesicle (GV) stage, CG located in the cortex; $B$, Oocyte at metaphase I stage with the formation of the first CG-free domain; $C$, Oocyte at metaphase II stage with a large second CG-free domain and partial CG exocytosis (arrowheads); $D$, Fertilized oocyte with full cortical granule exocytosis and a decondensing sperm head (arrow). Bar $=20 \mu \mathrm{m}$ for all panels.

hCG at day 6 of culture to induce final oocyte maturation (Figure 6B).

\section{Discussion}

We investigated the ability of $\mathrm{CM}$ to support in vitro 
follicular growth in organotypic culture and IVM of GV oocytes and subsequent embryo development. As expected, the results showed that two types of GV oocytes, which were isolated from 4-week-old mice $46 \mathrm{~h}$ after in vivo PMSG priming and cultured in CM, achieved a better meiotic competence compared with that in DMEM, $\alpha$ MEM, HTF, and a higher developmental competence than other culture media except $\alpha$-MEM. Furthermore, using CM supplemented with PMSG as culture medium, the follicles in ovarian organ cultured on soft agar grew well, and $60 \%$ of them matured to developmentally competent oocytes.

Previous studies with different species have all shown that the surrounding cumulus cells present in type $1 \mathrm{COCs}$ have an important role in the oocyte meiotic maturation
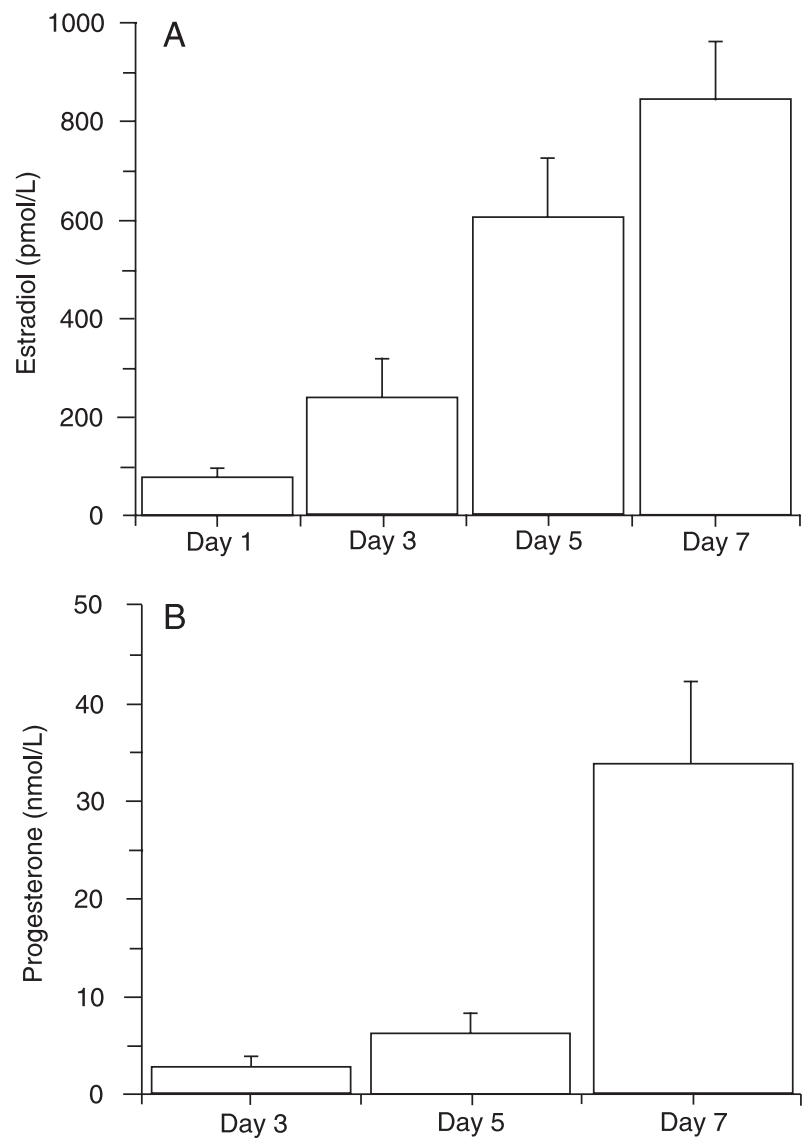

Figure 6. The hormone concentration in mesenchymal stem cell conditioned medium of in vitro cultured ovarian organ at different times. Each experiment was repeated six times. A, Estradiol concentration $(\mathrm{pmol} / \mathrm{L})$ progressively increased during culture. $B$, Production of progesterone $(\mathrm{nmol} / \mathrm{L})$ increased dramatically $17 \mathrm{~h}$ after stimulation with human chorionic gonadotropin at day 6 of culture. process $(16,17)$. This is probably mediated through their gap junction cytoplasmic connections to the ooplasm (18) that traverses the zona pellucida, as well as by the cumulus mass exerting a modulatory effect on the microenvironment surrounding the immature oocytes $(19,20)$. This could be important if the in vitro culture conditions are suboptimal. In our research, naked/partially naked oocytes (type 2) had reduced developmental competence after IVF compared with fully cumulus-enclosed oocyte complexes (type 1) after in vitro culture in DMEM, $\alpha-M E M$ and HTF for maturation. However, in contrast to maturation rate, there was no significant difference between the two categories of oocytes. Absence of cumulus cells and sequential insufficient cytoplasmic maturation may, at least in part, be responsible for this discrepancy. An unbalanced co-ordination of nuclear and cytoplasmic maturation was frequently observed in the IVM mouse oocytes $(21,22)$. In some IVM systems, cytoplasmic maturity was not fully achieved, which might cause reduced meiotic and developmental competence after fertilization $(3,23)$. Thus, improved meiotic maturation rate did not translate into any significant improvement in developmental competence, as assessed by IVF and blastocyst formation.

In contrast with the three culture media, when $\mathrm{CM}$ was used as maturation culture, excellent meiotic and developmental competence of IVM oocytes was achieved, irrespective of the presence of cumulus. The improvement of developmental competence was especially significant compared with DMEM, which was 2-fold at the two-cell stage and 12-fold at blastocyst formation. However, no significant difference existed between oocytes matured in $\mathrm{CM}$ and in vivo. We presume that the enriched cytokines and growth factors secreted by MSCs played the key role. Some of these secreted bioactive factors have been shown to improve meiotic maturation in vitro and subsequent embryo developmental potential directly or via cumulus cells (24-26). It has been reported that EGF and IGF-I enhance the rate of meiotic progression of bovine oocytes enclosed with cumulus cells $(8,27)$. Treatment with EGF during maturation culture decreased the time required for GVBD of denuded oocytes in mice (28) and EGF has a maturation-promoting effect on denuded bovine oocytes (29).

Oocyte maturation in vivo is a highly orchestrated, "induced" process, whereby the gonadotrophin/EGF cascade overrides the natural meiotic-inhibiting environment of the follicle (7). The EGF present in CM might reinitiate meiosis of oocyte maturation in vitro, similar to that which occurred in vivo and which prevented "spontaneous" nuclear maturation of IVM oocyte caused by removing the $\mathrm{COC}$ from the meiotic-inhibiting effects of the follicle. Cer- 
tainly, synchronous maturation of nuclear and cytoplasm in vitro led to a competent oocyte, which was confirmed by changes of CG distribution during IVM and subsequent embryonic development after IVF.

Changes in the distribution of CG during oocyte maturation have been used as an important criterion to evaluate cytoplasmic maturation $(30,31)$. Liu and colleagues (23) reported that mouse oocytes, matured under different conditions, differed in their ability to release CG upon activation. Culture media had marked effects on both nuclear progression and CG redistribution of IVM oocyte, and moreover significantly affected subsequent embryonic development. In the present study, temporal changes in the distribution of CG were observed in immature oocytes with or without cumulus cells cultured in CM. CGs were densely populated in a line mainly in the cortex at GV stage (Figure 5A). Typical normal CG-free domain formed at GVBD and MII stage (Figure 5B,C), and full-CG exocytosis occurred after IVF in sequentia (Figure 5D). The results showed that the process of nuclear maturation and $C G$ redistribution in oocytes matured in $\mathrm{CM}$ were similar to those matured in vivo as reported by Ducibella at al. (32). Using CG behavior as a marker for oocyte quality might provide guidance for the development of new IVM protocols.

The in vitro development of early preantral follicles is useful for understanding the mechanisms regulating the various stages of follicular growth and is of clinical importance. Many studies have been performed for the isolation of single follicles and their culture in vitro in rodents $(2,3)$. Two methods of enzymatic isolation and mechanical dissection were frequently used in previous studies $(3,33)$ to obtain follicles, which might cause unavoidable destruction to the microenvironment of follicles and damage to oocytes. In the present study, the sections of ovarian organ were cultured in vitro directly on soft agar allowing preantral follicles to develop. This model preserved the in vivo relationships of three types of cells (thecal cells, granulosa cells, and oocyte), and provided many kinds of exogenous growth factors secreted by MSCs, some of which were important components of follicle developmental microenvironment for appropriate "oocyte capacitation" or oocyte cytoplasmic development prior to maturation (7). Organotypic culture on soft agar prevented the follicles from attaching to the culture dish, which might lead to granulosa cell attachment and pristine outgrowth. In preantral follicles, granulosa cell culture was a necessity because an earlier outgrowth of granulosa cells resulted in oocyte release and cell degeneration most of the time (3). Furthermore, estrogen production in this model gave hormonal support for follicle growth. Moreover, we could deduce from the increasing amount of progesterone in response to the hCG stimulus that granulosa cells had, indeed, undergone normal differentiation. In this model, the oocytes retrieved at the end of culture matured sequentially in $\mathrm{CM}$, had an excellent developmental competence after IVF similar to that matured in vivo.

This report demonstrates that IVM oocytes cultured in $\mathrm{CM}$ can achieve a better meiotic competence than those cultured in DMEM, $\alpha$-MEM, HTF, and a higher developmental competence than other culture media except $\alpha$ MEM, while exhibited no significant difference compared with in vivo matured oocyte. $\mathrm{CM}$ can also support the preantral follicle growth in ovarian organ cultured on soft agar, which results in a satisfactory proportion of MII oocytes after maturation and an excellent developmental competence after IVF.

\section{Acknowledgments}

We thank Dr. Huai P. Zhu (University of Oklahoma, Health Sciences Center) and Dr. Gong L. Zhang (Florida Atlantic University) for helpful comments and critical reading of the manuscript. We also thank Mr. Xu Wu (School of Life Science, University of Science and Technology of China) for assistance with confocal microscopy experiments.

\section{References}

1. Miki H, Ogonuki N, Inoue K, Baba T, Ogura A. Improvement of cumulus-free oocyte maturation in vitro and its application to microinsemination with primary spermatocytes in mice. J Reprod Dev 2006; 52: 239-248.

2. Mousset-Simeon N, Jouannet $P$, Le Cointre L, Coussieu $C$, Poirot C. Comparison of three in vitro culture systems for maturation of early preantral mouse ovarian follicles. Zygote 2005; 13: 167-175.
3. Lenie S, Cortvrindt R, Adriaenssens T, Smitz J. A reproducible two-step culture system for isolated primary mouse ovarian follicles as single functional units. Biol Reprod 2004; 71: $1730-1738$.

4. Nakano $\mathrm{H}$, Kubo $\mathrm{H}$. Study of the in vitro maturation of mouse oocytes induced by microinjection of maturation promoting factor (MPF). J Assist Reprod Genet 2000; 17: 67-73.

5. Chin $\mathrm{AH}$, Chye NS. Investigations of oocyte in vitro matura- 
tion within a mouse model. Zygote 2004; 12: 1-18.

6. De La Fuente R, O'Brien MJ, Eppig JJ. Epidermal growth factor enhances preimplantation developmental competence of maturing mouse oocytes. Hum Reprod 1999; 14: 3060-3068.

7. Gilchrist RB, Thompson JG. Oocyte maturation: emerging concepts and technologies to improve developmental potential in vitro. Theriogenology 2007; 67: 6-15.

8. Sakaguchi M, Dominko T, Yamauchi N, Leibfried-Rutledge ML, Nagai T, First NL. Possible mechanism for acceleration of meiotic progression of bovine follicular oocytes by growth factors in vitro. Reproduction 2002; 123: 135-142.

9. Kuzmina TI, Alm H, Denisenko V, Tuchscherer A, Kanitz W, Torner $\mathrm{H}$. Effect of recombinant bovine somatotropin ( $r b S T)$ on cytoplasmic maturation of bovine oocytes and their developmental competence in vitro. J Reprod Dev 2007; 53: 309-316.

10. Trounson A, Anderiesz C, Jones G. Maturation of human oocytes in vitro and their developmental competence. Reproduction 2001; 121: 51-75.

11. Deans RJ, Moseley AB. Mesenchymal stem cells: biology and potential clinical uses. Exp Hematol 2000; 28: 875-884.

12. Caplan AI, Dennis JE. Mesenchymal stem cells as trophic mediators. J Cell Biochem 2006; 98: 1076-1084.

13. Kassem M, Kristiansen M, Abdallah BM. Mesenchymal stem cells: cell biology and potential use in therapy. Basic Clin Pharmacol Toxicol 2004; 95: 209-214.

14. Heil M, Ziegelhoeffer T, Mees B, Schaper W. A different outlook on the role of bone marrow stem cells in vascular growth: bone marrow delivers software not hardware. Circ Res 2004; 94: 573-574.

15. Miao YL, Liu XY, Qiao TW, Miao DQ, Luo MJ, Tan JH. Cumulus cells accelerate aging of mouse oocytes. Biol Reprod 2005; 73: 1025-1031.

16. Gilula NB, Epstein ML, Beers WH. Cell-to-cell communication and ovulation. A study of the cumulus-oocyte complex. J Cell Biol 1978; 78: 58-75.

17. Thompson JG. The impact of nutrition of the cumulus oocyte complex and embryo on subsequent development in ruminants. J Reprod Dev 2006; 52: 169-175.

18. Carabatsos MJ, Sellitto C, Goodenough DA, Albertini DF. Oocyte-granulosa cell heterologous gap junctions are required for the coordination of nuclear and cytoplasmic meiotic competence. Dev Biol 2000; 226: 167-179.

19. Byskov AG, Yding AC, Hossaini A, Guoliang X. Cumulus cells of oocyte-cumulus complexes secrete a meiosis-activating substance when stimulated with $\mathrm{FSH}$. Mol Reprod Dev 1997; 46: 296-305.

20. McNatty KP, Smith DM, Makris A, Osathanondh R, Ryan $\mathrm{KJ}$. Steroidogenesis by the human oocyte-cumulus cell complex in vitro. Steroids 1980; 35: 643-651.
21. Eppig JJ. Coordination of nuclear and cytoplasmic oocyte maturation in eutherian mammals. Reprod Fertil Dev 1996; 8: $485-489$

22. Sanfins A, Plancha CE, Overstrom EW, Albertini DF. Meiotic spindle morphogenesis in in vivo and in vitro matured mouse oocytes: insights into the relationship between nuclear and cytoplasmic quality. Hum Reprod 2004; 19: 28892899.

23. Liu XY, Mal SF, Miao DQ, Liu DJ, Bao S, Tan JH. Cortical granules behave differently in mouse oocytes matured under different conditions. Hum Reprod 2005; 20: 3402-3413.

24. Chian RC, Buckett WM, Tan SL. In-vitro maturation of human oocytes. Reprod Biomed Online 2004; 8: 148-166.

25. Martins da Silva SJ, Gardner JO, Taylor JE, Springbett A, De Sousa PA, Anderson RA. Brain-derived neurotrophic factor promotes bovine oocyte cytoplasmic competence for embryo development. Reproduction 2005; 129: 423-434.

26. Sutton ML, Gilchrist RB, Thompson JG. Effects of in-vivo and in-vitro environments on the metabolism of the cumulus-oocyte complex and its influence on oocyte developmental capacity. Hum Reprod Update 2003; 9: 35-48.

27. Sakaguchi $M$, Dominko $T$, Leibfried-Rutledge $M L$, Nagai $T$, First NL. A combination of EGF and IGF-I accelerates the progression of meiosis in bovine follicular oocytes in vitro and fetal calf serum neutralizes the acceleration effect. Theriogenology 2000; 54: 1327-1342.

28. Das K, Stout LE, Hensleigh HC, Tagatz GE, Phipps WR, Leung BS. Direct positive effect of epidermal growth factor on the cytoplasmic maturation of mouse and human oocytes. Fertil Steril 1991; 55: 1000-1004.

29. Lonergan $P$, Carolan C, Van Langendonckt A, Donnay I, Khatir $\mathrm{H}$, Mermillod $\mathrm{P}$. Role of epidermal growth factor in bovine oocyte maturation and preimplantation embryo development in vitro. Biol Reprod 1996; 54: 1420-1429.

30. Carneiro GF, Liu IK, Hyde D, Anderson GB, Lorenzo PL, Ball BA. Quantification and distribution of equine oocyte cortical granules during meiotic maturation and after activation. Mol Reprod Dev 2002; 63: 451-458.

31. Miyara F, Aubriot FX, Glissant A, Nathan C, Douard S, Stanovici A, et al. Multiparameter analysis of human oocytes at metaphase II stage after IVF failure in non-male infertility. Hum Reprod 2003; 18: 1494-1503.

32. Ducibella T, Kurasawa S, Rangarajan S, Kopf GS, Schultz RM. Precocious loss of cortical granules during mouse oocyte meiotic maturation and correlation with an egg-induced modification of the zona pellucida. Dev Biol 1990; 137: 4655.

33. Eppig JJ, O'Brien MJ. Development in vitro of mouse oocytes from primordial follicles. Biol Reprod 1996; 54: 197207. 